\title{
Mindfulness-based cognitive therapy versus psychoeducational intervention in bipolar outpatients with sub-threshold depressive symptoms: a randomized controlled trial
}

\author{
Guillermo Lahera', Carmen Bayón², Maria Fe Bravo-Ortiz², Beatriz Rodríguez-Vega², Sara Barbeito3, \\ Margarita Sáenz ${ }^{3}$, Caridad Avedillo², Rosa Villanueva², Amaia Ugarte ${ }^{3}$, Ana González-Pinto ${ }^{3}$ and Consuelo de Dios²*
}

\begin{abstract}
Background: The presence of depressive subsyndromal symptoms (SS) in bipolar disorder (BD) increases the risk of affective relapse and worsens social, cognitive functioning, and quality of life. Nonetheless, there are limited data on how to optimize the treatment of subthreshold depressive symptoms in BD. Mindfulness-Based Cognitive Therapy (MBCT) is a psychotherapeutic intervention that has been shown effective in unipolar depression. The assessment of its clinical effectiveness and its impact on biomarkers in bipolar disorder patients with subsyndromal depressive symptoms and psychopharmacological treatment is needed.
\end{abstract}

Methods/design: A randomized, multicenter, prospective, versus active comparator, evaluator-blinded clinical trial is proposed. Patients with BD and subclinical or mild depressive symptoms will be randomly allocated to: 1) MBCT added to psychopharmacological treatment; 2) a brief structured group psychoeducational intervention added to psychopharmacological treatment; 3) standard clinical management, including psychopharmacological treatment. Assessments will be conducted at screening, baseline, post-intervention (8 weeks) and 4 month follow-up post-intervention. The aim is to compare MBCT intervention versus a brief structured group psychoeducation. Our hypothesis is that MBCT will be more effective in reducing the subsyndromal depressive symptoms and will improve cognitive performance to a higher degree than the psychoeducational treatment. It is also hypothesized that a significant increase of BDNF levels will be found after the MBCT intervention.

Discussion: This is the first randomized controlled trial to evaluate the effects of $\mathrm{MBCT}$ compared to an active control group on depressive subthreshold depressive symptoms in patients with bipolar disorder.

Trial registration: ClinicalTrials.gov: NCT02133170. Registered 04/30/2014.

Keywords: Mindfulness, Psychoeducation, Bipolar disorder, Depression, Subsyndromal symptoms, Meditation, BDNF, Randomized clinical trial

\section{Background}

Even in environments with standard psychiatric treatment and regular follow-up, around 38\% of patients who have recovered from an acute episode of bipolar disorder (BD) show persistent subthreshold symptoms (SS) [1]. The Depression Comorbidity Study shows that the time with SS is higher than time in episode throughout the

\footnotetext{
* Correspondence: consuelo.dios@salud.madrid.org

${ }^{2}$ University Hospital La Paz, IDIPAZ, Madrid, Spain

Full list of author information is available at the end of the article
}

follow-up, with a clear predominance of depressive SS [2]. In a Spanish cohort, patients were symptomatic for one third of the 72-weeks follow-up, and also one third of the visits. In addition, they spent three times more days depressed than manic or hypomanic [3]. The persistence of SS has been associated with an increased risk of affective relapse/recurrence [4-6], a shorter time to relapse [7], greater functional impairment-similar to that experienced by patients with depressive episode-,

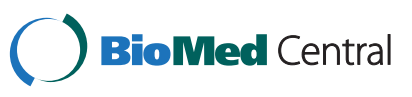

(c) 2014 Lahera et al.; licensee BioMed Central Ltd. This is an Open Access article distributed under the terms of the Creative Commons Attribution License (http://creativecommons.org/licenses/by/4.0), which permits unrestricted use, distribution, and reproduction in any medium, provided the original work is properly credited. The Creative Commons Public Domain Dedication waiver (http://creativecommons.org/publicdomain/zero/1.0/) applies to the data made available in this article, unless otherwise stated. 
worse quality of life, delayed functional recovery $[8,9]$ ) and poorer cognitive performance [10].

Nevertheless, few studies have assessed the efficacy of treatments on bipolar patients with depressive SS. Evidence of effectiveness using only drugs is limited, therefore alternatives are needed. Concomitant psychosocial interventions are recommended, such cognitive behavioral therapy (CBT), interpersonal and social rhythm therapy (IPSRT) and structured psychoeducation, especially for stabilization between episodes and relapse prevention [11]. In a recent study, a 21-session individual CBT was shown as effective -but much less cost-effectiveas a brief psychoeducational group intervention, with overall decrease in disease burden, effectiveness in preventing episodes and time to relapse [12]. A combined treatment (pharmacological plus psychoeducational and cognitive-behavioral therapy) has shown to decrease depressive symptoms, even in refractory $\mathrm{BD}$, and the number of hospitalizations [13]. Despite psychoeducational interventions appear to be most promising, the development of effective psychosocial therapies is still needed.

Mindfulness and acceptance-based interventions (MABIs) are receiving increasing attention in the treatment of mental disorders and show promise for symptoms of anxiety and depression both in clinical and nonclinical populations [14]. The practice of mindfulness works well in cognitive processes (rumination, cognitive reactivity, experiential avoidance), which have been associated with vulnerability to depressive and anxious symptoms and neuropsychological performance [15]. Mindfulness is currently defined in psychological terms as "paying total attention to the present moment with a non-judgmental awareness of inner and outer experiences" [16]. The primary mechanism of action is the moment-by-moment attention of our thoughts, feelings, bodily sensations, and surrounding environment, which promotes emotional regulation and awareness of the basic processes influencing the attribution of meaning to experiences. As important as the attention training is the promotion of an attitude of openness, curiosity and acceptance of all the experiences that arise. MindfulnessBased Cognitive Therapy (MBCT), developed by Segal, Williams and Teasdale [17] is a group-based, 8-week, mind-body intervention that integrates psychological educational aspects of CBT for depression with meditation components of mindfulness-based stress reduction developed by Kabat-Zinn [16]). It has proven effective as adjunctive therapy in preventing relapse of recurrent depressive disorder [18-21] and reducing the residual depressive symptoms in unipolar depression [22,23]. In a pilot trial, MBCT compared to psychoeducation was significantly more effective in improving subsyndromal depressive symptoms in patients with unipolar depression [24]. In addition, MBCT is recommended in the NICE (National Institute for Health and Clinical Excellence) clinical guideline for the treatment of unipolar depressive patients with a history of 3 or more episodes [25].

Some preliminary studies and a randomized controlled trial with a long follow-up have examined MBCT in the treatment for bipolar disorder. Williams et al. [26] conducted a pilot clinical trial evaluating the effectiveness of MBCT versus waitlist on between-episode anxiety and depressive symptoms. Although the results were favorable for MBCT, these should be analyzed with caution given the small size and heterogeneity of the sample. Miklowitz et al. [27] studied the effect of a modified MBCT protocol with two additional treatment elements specifically designed for patients with bipolar disorder: psychoeducation about mood changes and its provoking factors (e.g. interpersonal conflict, sleep/wake cycle disruptions) and upon identification of these factors bringing mindfulness to prodromal signs of mood elevation. Small to medium effect sizes for improvement in depression and anxiety symptoms in remitted patients with bipolar disorder were found. In other preliminary study, at the end of a MBCT treatment, as well as at the 3-month follow-up, participants with BD showed lower residual depressive mood symptoms, less attentional difficulties, increased emotion-regulation abilities, psychological wellbeing, more positive affect, and psychosocial functioning and improvements in executive functioning, memory, and ability to initiate and complete tasks [28]. Other controlled studies have also shown a beneficial effect of MBCT for patients with BD, but with limitations such as small sample, absence of randomization or of an active comparator $[29,30]$. Perich et al. [31] have compared MBCT plus treatment as usual (TAU) to TAU alone in a RCT over a 12 month follow-up period. MBCT did not lead to significant reductions in time to depressive or hypo/manic relapse, total number of episodes, or mood symptom severity at 12-month follow-up; however, there was some evidence for an effect on anxiety symptoms. Despite decreases in depression were only observed at a trend level, further research is required to analyze the effect of MBCT on residual depressive symptoms in bipolar patients.

Mindfulness-based interventions are cost-effective, showing a potential to significantly reduce societal costs and increase the effectiveness of care [32]. Research in this field has shown important methodological limitations: differences in the application of the MBCT intervention, both in duration and number of sessions or the amount of incorporated psychoeducational elements; the limited statistical power due to small sample size; lack of control for possible confounding factors such as pharmacological treatment, or lack of information on the severity of the disease; and scarcity of long-term randomized clinical trials that allow to assess the maintenance of achieved effects. In the present project we try to overcome several of these limitations, 
especially by using the method of randomized clinical trial with an active comparator, the larger sample size and the attempt to control confounding variables regarding the condition and design of the intervention.

Structured group psychoeducation is an adjunctive psychological treatment that has been shown to be effective in the prevention of affective recurrences as well as number of hospitalizations and time with symptoms in BD euthymic outpatients, both at two-year [33] and five-year follow-up [34]. This psychoeducational program is structured in 21 sessions delivered through 6 months. It stresses the importance of illness awareness, self-management, early-warning sign identification, habit regularity, treatment adherence and avoiding drug misuse. "It has been defined as "behavioural psychoeducation", but its authors rather think of it as an "attitudes \& aptitudes" psychoeducational program. This definitely corresponded to a view of bipolar disorder as a complex condition involving not only biological etiological factors but also psychological and social variables that may act as triggering factors, modulators or mediators" [35]. This study has been successfully replicated in pharmacologically treated patients with bipolar disorder also in routine clinical settings [36].

The brain -derived neurotrophic factor (BDNF) is a dimeric protein that promotes the growth and maintenance of neuronal connections, modulates neurotransmission and participates in the mechanisms of learning and longterm plasticity (LTP). Peripheral BDNF in serum and plasma can be assessed noninvasively by venipuncture. Since it crosses the blood-brain barrier, its levels in serum and plasma are highly correlated with levels in cerebrospinal fluid ( $\mathrm{r}^{1 / 4}$ 0.8) $[37,38]$. There are numerous studies assessing peripheral levels of BDNF in bipolar disorder patients. Some studies suggest that BDNF levels decrease during mood acute states and remain normal during euthymia, but other studies have contradicted this paradigm. A recent meta-analysis has measured the effect sizes of the differences in BDNF levels between BD patients in different mood states and controls. The BDNF levels were decreased in both mania and depression when compared to controls $[39,40]$ but were not different in euthymia [41]. Meta-regression analyses in euthymia showed that age and length of illness influenced the variation in effect sizes [42]). BDNF levels are consistently reduced during manic and depressive episodes and recover after treatment for acute mania, so peripheral BDNF could be used as a biomarker of mood states and disease progression for $\mathrm{BD}$.

In summary, the presence of persistent depressive subthreshold symptoms increases the risk of affective relapse and negatively affects the prognosis of BD. However, there is no an evidence-based specific treatment for them. $\mathrm{MBCT}$ appears as a promising intervention, since it has been found to be effective in reducing current depressive symptoms in MDD [23,43]. Our objective is to evaluate the efficacy of the MBCT added to standard drug treatment -following the recommendations of the Spanish Guideline for the Treatment of BD [11])- versus structured group psychoeducation versus standard clinical management. Our primary outcome is reduction of residual depressive symptoms. Secondary outcomes are manic and anxiety symptoms, recurrence, cognitive and social functioning and levels of BDNF.

\section{Methods/design}

This is a parallel 3-group, multicenter, prospective, randomized; single-blind (evaluator) controlled pilot trial, with a 4 month follow-up post-intervention. Patients diagnosed with bipolar disorder (BD) according to DSM -5 criteria for mild depression or subsyndromal depressive symptoms are assigned to one of the following 3 treatment groups: 1) psychopharmacological treatment plus Mindfulness Based Cognitive Therapy (MBCT); 2) psychopharmacological treatment plus a brief, structured group psychoeducation; 3) treatment as usual (TAU), defined as standard psychiatric care with standard pharmacologic treatment. After written informed consent is signed, patients meeting the inclusion criteria are randomized (2:2:1 ratio) through a Random Allocation Software. All three groups are assessed at baseline ( $\mathrm{t} 0$ ), immediately after completing the program ( $\mathrm{t} 1 ; 8$ weeks) and at follow-up six months after randomization. The assessments include the following variables: depression, manic and anxiety symptoms, general and social cognition, global functioning, BDNF, and other clinical variables. The evaluator who will collect the biomarkers and the clinical and psychometric data will be blind to allocation of treatment. The interrater variability between all researchers will be checked.

\section{Subjects}

One hundred and forty bipolar out-patients with persistent mild/subsyndromal depressive symptoms will be recruited from Mental Health Centers as well as Private Psychiatric Clinics and Bipolar Patient Associations via announcements or physician referral. The study will be conducted in La Paz University Hospital (Madrid) and the Santiago Apóstol University Hospital (Vitoria).

\section{Inclusion criteria}

1-Age: 18-60 years; 2 - BD type I or II, in clinical remission of acute mood episode at least in the three months prior to study; 3 - Having experienced an acute affective episode in the past 3 years, 4 - Having suffered at least two lifetime depressive episodes. 5- Monotherapy or combination with a mood stabilizer (lithium, 
valproate, carbamazepine or lamotrigine) at optimal doses (i.e., in serum levels within the therapeutic range: 0.6$1.2 \mathrm{mEq} / \mathrm{L}$ for lithium, $50-100 \mathrm{ug} / \mathrm{mL}$ for valproate, and 5-12 $\mathrm{mcg} / \mathrm{mL}$ for carbamazepine), or quetiapine monotherapy or in combination with the aforementioned stabilizers, or any oral atypical antipsychotic in combination with an antidepressant; 6.- Hamilton Depression Rating Scale [HDRS] -17 [44] score $\geq 8$ and $\leq 19$ and Young Mania Rating Scale [YMRS; 49] score $<8$; 7 - Being able to understand and comply with the requirements of the trial, 8 Written consent to participate in the study.

\section{Exclusion criteria}

1. Any acute mood episode in the 12 weeks before the start of the trial. 2. Any current DSM -5 diagnosis different from bipolar disorder (including substance or alcohol use disorder at the time of study entry, except if it is under complete remission. Not applicable to nicotine or caffeine). 3. Risk of suicide or self/hetero aggressiveness. 4. Pregnancy. 5. Severe and unstable medical disease. 6. Patients who are currently receiving structured psychotherapy or structured group psychoeducation about bipolar disorder, or who have received structured psychoeducation in the past 5 years; 6 . Patients who are treated with a different mood stabilizer than lithium, valproate, carbamazepine, lamotrigine, a classic antipsychotic or antidepressant monotherapy at the time of the randomization. 7. Treatment with a depot antipsychotic; 8. Participation in another clinical trial within 4 weeks prior to randomization. 9. Mental retardation.

\section{Withdrawal criteria}

Express wish of the participant, unjustified absence from more than two group training sessions, appearance of suicidal ideation or psychotic symptoms, onset of acute manic or hypomanic episode, or need for psychiatric hospitalization from any cause.

\section{Primary outcome measures}

Primary endpoint of the study is given by changes in the overall score of the Hamilton Rating Scale for Depression (HDRS [44]), from baseline (V0) to week 8 (v1) for each of the treatment groups.

\section{Secondary endpoints}

1. Changes in the global score of Young Mania Rating Scale (YMRS, [45]) from baseline (V0) to visit 1 (at the end of the 8 week intervention (v1)

2. Changes in the score of Clinical Global Impression CGI-BP [46] from baseline (V0) to visit 1;

3. Changes in the score of the Hamilton Rating Scale for Anxiety HAM-A [47] from baseline (V0) to visit 1 ;
4. Cognitive changes: changes at the end of the intervention will be assessed with the following measures:

- sustained and selective attention

- working memory and executive functions

- perception of the attitude of mindfulness (Five Facet Mindfulness Questionnaire FFMQ, [48]) in patients in the experimental group

- scales of social cognition

5. Functioning: changes in total scale score of the

Functioning Assessment Short Test (FAST; [49]).

6. The following clinical variables are also assessed:

- Recurrence, defined as the emergence of a new acute episode whether depressive, mixed, hypo or manic at any time throughout the study, according to DSM- 5 clinical criteria or when the score on the HDRS scale is $\geq 20$ (depressive episode) or the Young scale ( YMRS $\geq 8$ ) (hypo/ manic episode), or a change drug or hospitalization is needed.

- Plasma levels of BDNF: changes from baseline to visit 1 (end of intervention).

Twenty-four weeks after the start of intervention following measurements are assessed: changes in the overall score of the Hamilton Depression Rating Scale (HDRS [44]), Young Mania Rating Scale (YMRS, [45]) from baseline (V0) to week 24 (V2), Hamilton anxiety Scale HAM- A [47], CGI [46], general cognitive functioning, social cognition, functioning and biomarkers (BDNF).

\section{Intervention}

MBCT program will be conducted in HULP by two experienced therapists with recognized expertise in Mindfulness based stress reduction (MBSR), MBCT and narrative psychotherapy. Both therapists will train two therapists from Vitoria with expertise in cognitive therapy and MBSR. The MBCT program consists of 8 weekly sessions of 90 minutes and will be performed in groups of approximately $10-15$ patients. All therapy sessions will be audio recorded to be discussed and analyzed by the treatment team, in order to standardize the intervention. Treatment fidelity will be also assessed using the Mindfulness- based Cognitive Therapy Adherence Scale (MBCT-AS; [50]), which assesses the key constructs of MBCT during group sessions.

Psychoeducation program will be held in groups of 10 to 15 patients in 90 -minute weekly sessions led by two therapists blind to the clinical and cognitive evaluation. The specific program of 8 sessions addresses disease awareness, adherence to treatment and early detection of prodromal symptoms. Homework will also be included. The program is based on the Psychoeducation Manual for 
Bipolar Disorder [51]. Attendance to at least $80 \%$ of the sessions of both interventions will be required to be considered complete.

\section{Assessment instruments a) Clinical variables}

1 Depressive symptoms: measured by the Hamilton Depression Rating Scale of 17 items (HDRS-17) [44,52]. 3. Manic symptoms: measured by the Young Mania Rating Scale (YMRS) [45]. 4. Hamilton Anxiety Scale HAM-A [47]. 5. Clinical Global Impression Scale modified for bipolar disorder (CGI-BP) [46]. 6. Adherence to treatment: Good (according to information from the patient, family and blood drug levels), Bad (if none of the above criteria are met) or Average (only if it meets one or two criteria). 7. Clinical outcome: new mood episodes, serious adverse events (including suicidal ideation, suicide and hospitalization).

\section{b) Cognitive variables}

\section{General cognition}

- Sustained attention (Continuous Performance Test, [53]). The test provides the following measures: correct responses, errors of omission (not responding when the stimulus appears) and commission errors (responding without the letter stimulus appearance).

- Selective attention (Stroop Test, [48]). Requires the subject to be able to suppress irrelevant response. When the name of a color (e.g., "blue," "green," or "red") is printed in a color not denoted by the name (e.g., the word "red" printed in blue ink instead of red ink), naming the color of the word takes longer and is more prone to errors than when the color of the ink matches the name of the color [54].

- Working memory. 1. Subtest from the Weschler Adult Intelligence Scale [55]. A sequence from 2 to 8 digits or a list of words have to be repeated in reverse or random order of presentation.

- Executive functions: 1. Wisconsin Card Sorting Task [56]. Participants have to sort the cards according to different criteria that vary throughout the test (perseverative errors and completed categories are the dependent variables).

\section{c) Social cognition}

The recognition of emotions through the face is measured with the Face Emotion Identification Task and Face Emotion Discrimination Task (FEIT and FEDT respectively, [57]). The FET scale includes 19 photographs of faces expressing one of the six basic emotions (happy, sad, angry, scared, shocked or embarrassed). The task is to identify the patient's emotion expressed in each. The FEDT includes 30 pairs of faces and the subject must determine if both sides show the same or different emotion.

\section{d) Mindfulness}

Five Factors Questionnaire Mindfulness (FFMQ, [58]) 39 items assessing five mindfulness facets: observing, describing, acting with awareness, equanimity and no reactivity. Each item is scored on a Likert scale ranging from 1 (never) to 5 (very often). A high internal consistency and high predictive validity have been shown [58] and it has been uvalidated in Spanish [59].

\section{e) Functioning}

Measured with the Functioning Assessment Short Test (FAST, [49]), which measures the degree of difficulty found by the patient in the following areas: autonomy, occupational functioning, cognitive functioning, finances, relationships and leisure.

\section{f) Biomarkers: serum analysis of BDNF}

\section{Data analysis}

An exploratory study of all collected variables identifies possible outliers and errors. The descriptive analyses of qualitative variables are expressed as frequencies and percentages and quantitative variables as mean, standard deviation, median and interquartile range. Analysis is performed by intention to treat. Comparison of qualitative variables between the intervention groups or between other factors of segmentation is by means of Chi 2 or Fisher 's exact test. For quantitative variables, the Ttest, Mann -Whitney or Kruskal -Wallis and ANOVA are applied, depending on the normality of the variable (tested by Kolmogorov -Smirnov - Lilliefords). The change analyses along the track of the primary and secondary outcome variables are performed using a General Linear Model (ANOVA with repeated measures). Regression models are extracted to identify prognostic factors for the efficacy variables. The statistical package SPSS 18.0 is used in all tests and a bilateral level of $\mathrm{p}<0.05$ is considered statistically significant.

\section{Ethical issues}

The study is conducted according to local regulations and internationally established principles at the the Helsinki Statement (64 Asamblea General Fortaleza, Brasil, 2013), whilst respecting confidentiality (Data Protection Act 15/99). Protocol was approved by the Clinical Research Ethics Committee of the University Hospital La Paz (Madrid) and the Clinical Research Ethics Committee of the Basque Country. 


\section{Discussion}

This study will provide feasibility information and preliminary effect size estimates to inform the effectiveness of adjunctive $\mathrm{MBCT}$, adjunctive group psychoeducation and standard treatment on SS or mild depressive symptoms in BD. It is of interest to researchers involved in the development of new approaches to the treatment of residual affective symptoms and its negative impact in the course of illness. It can also provide information on variations of BDNF in the different treatment arms and on neuropsychological measures, which might suggest mechanisms of action and differences among treatments.

Strengths of the study include targeting a defined sample of bipolar patients with persistent depressive symptoms despite receiving a correct pharmacological treatment. This sample -as reported before- is representative of a high percentage of bipolar out-patients, who remain symptomatic more than one third of the time. The trial protocol was registered before randomization began (ClinicalTrials.gov: NCT02133170 Registered 04/30/2014) and was developed according to good clinical research practice enabling the randomized trial to be conducted with low risk of bias and a high degree of external validity. Both MBCT and psychoeducational interventions will be conducted using manuals and adherence to the treatment manuals will be tested. This makes it possible to implement the interventions in clinical practice, and the two interventions can be assessed in future trials. To standardize the application of MBCT and psychoeducation, specific training and meetings will be held. Specific training also may increase the inter-rater reliability on scales.

Since an 8-week psychoeducational program based in the Barcelona group-structured model has not proved effective neither in depressive symptoms nor in episode prevention, this could be a study weakness. But our objective is to compare MBCT versus an active psychosocial intervention, and this is the best model we could find. Sample size could also be a limitation, as well as the time of follow-up, especially regarding the possible effects on long-term course of BD.

\begin{abstract}
Abbreviations
ANOVA: Analysis of variance; BD: Bipolar disorder; BDNF: Brain derived neurotrophic factor; CBT: Cognitive behavioral therapy; CGI-BP: Clinical global impression scale modified for bipolar disorder; FAST: Functioning assessment short test; FEDT: Face emotion discrimination task; FEIT: Face emotion identification task; FFMQ: Five factors questionnaire mindfulness; HAM-A: Hamilton anxiety scale HAM-A; HDRS: Hamilton depression rating scale; IPSRT: Interpersonal and social rhythm Therapy; ABls: Mindfulness and acceptance-based interventions; MBCT: Mindfulness based cognitive therapy; SS: Subsyndromal symptoms; YMRS: Young mania rating scale.
\end{abstract}

\section{Competing interests}

The authors declare that they have no competing interests.

\section{Authors' contributions}

All authors have made substantial contributions to this study protocol. CD is the Principal Investigator of the study. CD and GL conceived of the design of the study and participated in the design of the intervention, the development of the statistical analysis plan, and had overall responsibility for the study. CB, $\mathrm{BR}$ and $\mathrm{MB}$ helped with the study design and analysis plan, participated in the coordination of the trial and the implementation of the intervention. All authors helped to draft the manuscript, read and approved the final version.

\section{Acknowledgements}

This work was supported in part by the Spanish grant FIS P/13/00352 from Instituto de Salud Carlos III.

\section{Author details}

${ }^{1}$ Department of Medicine and Medical Specialties, University of Alcalá, Madrid, Spain. ${ }^{2}$ University Hospital La Paz, IDIPAZ, Madrid, Spain. ${ }^{3}$ CIBERSAM Biomedical Research Centre in Mental Health Net (CIBERSAM), University Hospital of Álava, Vitoria-Gasteiz, Spain.

Received: 6 July 2014 Accepted: 18 July 2014

Published: 15 August 2014

\section{References}

1. Tohen M, Bowden CL, Calabrese JR, Lin D, Forrester TD, Sachs GS, Koukopoulos A, Yatham L, Grunze H: Influence of sub-syndromal symptoms after remission from manic or mixed episodes. $\mathrm{Br} J$ Psychiatry 2006, 189:515-519.

2. Judd LL, Akiskal HS, Schettler PJ, Endicott J, Maser J, Solomon DA, Leon AC, Rice JA, Keller MB: The long-term natural history of the weekly symptomatic status of bipolar I disorder. Arch Gen Psychiatry 2002, 59(6):530-537.

3. De Dios C, Ezquiaga E, Garcia A, Soler B, Vieta E: Time spent with symptoms in a cohort of bipolar disorder outpatients in Spain: a prospective, 18-month follow-up study. J Affect Disord 2010, 125(1-3):74-81.

4. Goodnick PJ, Fieve RR, Schlegel A, Baxter N: Predictors of interepisode symptoms and relapse in affective disorder patients treated with lithium carbonate. Am J Psychiatry 1987, 144(3):367-369.

5. Keller MB, Lavori PW, Kane JM, Gelenberg AJ, Rosenbaum JF, Walzer EA, Baker LA: Subsyndromal symptoms in bipolar disorder: a comparison of standard and low serum levels of lithium. Arch Gen Psychiatry 1992 49(5):371-376.

6. Kleindienst N, Greil W: Inter-episodic morbidity and drop-out under carbamazepine and lithium in the maintenance treatment of bipolar disorder. Psychol Med 2002, 32(3):493-501.

7. De Dios C, Ezquiaga E, Agud JL, Vieta E, Soler B, García-López A: Subthreshold symptoms and time to relapse/recurrence in a community cohort of bipolar disorder outpatients. J Affect Disord 2012, 143(1-3):160-165.

8. Gutiérrez-Rojas L, Gurpegui M, Ayuso-Mateos JL, Gutiérrez-Ariza JA, Ruiz-Veguilla M, Jurado D: Quality of life in bipolar disorder patients: a comparison with a general population sample. Bipolar Disord 2008, 10(5):625-634.

9. Bonnín CM, Sánchez-Moreno J, Martínez-Arán A, Solé B, Reinares M, Rosa AR, Goikolea JM, Benabarre A, Ayuso-Mateos JL, Ferrer M, Vieta E, Torrent C: Subthreshold symptoms in bipolar disorder: impact on neurocognition, quality of life and disability. J Affect Disord 2012, 136(3):650-659.

10. Bonnín CM, Martínez-Arán A, Torrent C, Pacchiarotti I, Rosa AR, Franco C, Murru A, Sanchez-Moreno J, Vieta E: Clinical and neurocognitive predictors of functional outcome in bipolar euthymic patients: a long-term, follow-up study. J Affect Disord 2010, 121(1-2):156-160.

11. Bravo MF, Lahera G, Lalucat $L$, Fernández-Liria A: Clinical practice guideline on bipolar disorder: drug and psychosocial therapy. Asociación Española de Neuropsiquiatría. Med Clin (Barc) 2013, 141(7):305.

12. Parikh SV, Zaretsky A, Beaulieu S, Yatham LN, Young LT, Patelis-Siotis I, Macqueen GM, Levitt A, Arenovich T, Cervantes P, Velyvis V, Kennedy SH, Streiner DL: A randomized controlled trial of psychoeducation or cognitive-behavioral therapy in bipolar disorder: a Canadian Network for Mood and Anxiety treatments(CANMAT) study [CME]. J Clin Psychiatry 2012, 73(6):803-810.

13. González-Isasi A, Echeburúa E, Mosquera F, Ibáñez B, Aizpuru F, González-Pinto A: Long-term efficacy of a psychological intervention program for patients with refractory bipolar disorder: a pilot study. Psychiatry Res 2010, 176(2-3):161-165. 
14. Vøllestad J, Nielsen MB, Nielsen GH: Mindfulness- and acceptance-based interventions for anxiety disorders: a systematic review and meta-analysis. Br J Clin Psychol 2012, 51(3):239-260.

15. Chiesa A, Serretti A: Mindfulness based cognitive therapy for psychiatric disorders: a systematic review and meta-analysis. Psychiatry Res 2011, 187(3):441-453.

16. Kabat-Zinn J: Full Catastrophe Living: How to Cope with Stress, Pain and Illness Using Mindfulness Meditation. New York: Delacorte; 1990.

17. Segal Z, Williams M, Teasdale J: Mindfulness-based Cognitive Therapy for Depression: A New Approach to Preventing Relapse. New York: Guilford Press; 2002.

18. Teasdale JD, Segal ZV, Williams JM, Ridgeway VA, Soulsby JM, Lau MA: Prevention of relapse/recurrence in major depression by mindfulness-based cognitive therapy. J Consult Clin Psychol 2000, 68(4):615-623.

19. Ma SH, Teasdale JD: Mindfulness-based cognitive therapy for depression: replication and exploration of differential relapse prevention effects. J Consult Clin Psychol 2004, 72(1):31-40.

20. Bondolfi G, Jermann F, der Linden MV, Gex-Fabry M, Bizzini L, Rouget BW, Myers-Arrazola L, Gonzalez C, Segal Z, Aubry JM, Bertschy G: Depression relapse prophylaxis with mindfulness-based cognitive therapy: replication and extension in the Swiss health care system. J Affect Disord 2010, 122(3):224-231

21. Piet J, Hougaard E, Hecksher MS, Rosenberg NK: A randomized pilot study of mindfulness-based cognitive therapy and group cognitivebehavioral therapy for young adults with social phobia. Scand J Psychol 2010, 51(5):403-410.

22. Kingston T, Dooley B, Bates A, Lawlor E, Malone K: Mindfulness-based cognitive therapy for residual depressive symptoms. Psychol Psychother 2007, 80(Pt 2):193-203.

23. Barnhofer T, Crane C, Hargus E, Amarasinghe M, Winder R, Williams JMG: Mindfulness-based cognitive therapy as a treatment for chronic depression: a preliminary study. Behav Res Ther 2009, 47:366-373.

24. Chiesa A, Mandelli L, Serretti A: Mindfulness-based cognitive therapy versus psycho-education for patients with major depression who did not achieve remission following antidepressant treatment: a preliminary analysis. J Altern Complement Med 2012, 18(8):756-760.

25. NICE: Depression: Treatment and Management of Depression in Adults. Clinical Guideline 90. London: National Institute for Health and Clinical Excellence; 2009. Available at www.nice.org.uk.

26. Williams JM, Alatiq Y, Crane C, Barnhofer T, Fennell MJ, Duggan DS, Hepburn S, Goodwin GM: Mindfulness-based Cognitive Therapy $(\mathrm{MBCT})$ in bipolar disorder: preliminary evaluation of immediate effects on between-episode functioning. J Affect Disord 2008, 107(1-3):275-279.

27. Miklowitz DJ: Functional impairment, stress, and psychosocial intervention in bipolar disorder. Curr Psychiatry Rep 2011, 13(6):504-512.

28. Stange JP, Eisner LR, Hölzel BK, Peckham AD, Dougherty DD, Rauch SL, Nierenberg AA, Lazar S, Deckersbach T: Mindfulness-based cognitive therapy for bipolar disorder: effects on cognitive functioning. J Psychiatr Pract 2011, 17(6):410-419.

29. Weber B, Jermann F, Gex-Fabry M, Nallet A, Bondolfi G, Aubry JM: Mindfulnessbased cognitive therapy for bipolar disorder: a feasibility trial. Eur Psychiatry 2010, 25(6):334-337.

30. Deckersbach T, Hölzel BK, Eisner LR, Stange JP, Peckham AD, Dougherty DD, Rauch SL, Lazar S, Nierenberg AA: Mindfulness-based cognitive therapy for nonremitted patients with bipolar disorder. CNS Neurosci Ther 2012 18(2):133-141

31. Perich T, Manicavasagar V, Mitchell PB, Ball JR, Hadzi-Pavlovic D: A randomized controlled trial of mindfulness-based cognitive therapy for bipolar disorder. Acta Psychiatr Scand 2013, 127(5):333-343.

32. Fjorback LO, Carstensen T, Arendt M, Ornbøl E, Walach H, Rehfeld E, Fink P. Mindfulness therapy for somatization disorder and functional somatic syndromes: analysis of economic consequences alongside a randomized trial. J Psychosom Res 2013, 74(1):41-48.

33. Colom F, Vieta E, Martinez-Aran A, Reinares M, Goikolea JM, Benabarre A, Torrent C, Comes M, Corbella B, Parramon G, Corominas J: A randomized trial on the efficacy of group psychoeducation in the prophylaxis of recurrences in bipolar patients whose disease is in remission. Arch Gen Psychiatry 2003, 60(4):402-407.

34. Colom F, Vieta E, Sánchez-Moreno J, Palomino-Otiniano R, Reinares M, Goikolea JM, Benabarre A, Martínez-Arán A: Group psychoeducation for stabilised bipolar disorders: 5-year outcome of a randomised clinical trial. Br J Psychiatry 2009, 194(3):260-265.

35. Colom F: The evolution of psychoeducation for bipolar disorder: from lithium clinics to integrative psychoeducation. World Psychiatr 2014, 13(1):90-92.

36. Candini V, Buizza C, Ferrari C, Caldera MT, Ermentini R, Ghilardi A, Nobili G, Pioli R, Sabaudo M, Sacchetti E, Saviotti FM, Seggioli G, Zanini A, de Girolamo G: Is structured group psychoeducation for bipolar patients effective in ordinary mental health services? A controlled trial in Italy. J Affect Disord 2013, 151(1):149-155.

37. Karege F, Bondolfi G, Gervasoni N, Schwald M, Aubry JM, Bertschy G: Low brainderived neurotrophic factor (BDNF) levels in serum of depressed patients probably results from lowered platelet BDNF release unrelated to platelet reactivity. Biol Psychiatry 2005, 57(9):1068-72.

38. Pan W, Banks WA, Fasold MB, Bluth J, Kastin AJ: Transport of brainderivedneurotrophic factor across the blood-brain barrier. Neuropharmacology 1998, 37(12):1553e61.

39. Tramontina J, Frey BN, Andreazza AC, Zandona M, Santin A, Kapczinski F: Val66met polymorphism and serum brain-derived neurotrophic factor levels in bipolar disorder. Mol Psychiatry 2007, 12(3):230-231.

40. de Oliveira GS, Ceresér KM, Fernandes BS, Kauer-Sant'Anna M, Fries GR, Stertz L, Aguiar B, Pfaffenseller B, Kapczinski F: Decreased brain-derived neurotrophic factor in medicated and drug-free bipolar patients. J Psychiatr Res 2009, 43(14):1171-1174

41. Cunha AB, Frey BN, Andreazza AC, Goi JD, Rosa AR, Gonçalves CA, Santin A, Kapczinski F: Serum brain-derived neurotrophic factor is decreased in bipolar disorder during depressive and manic episodes. Neurosci Lett 2006, 398(3):215-219.

42. Fernandes BS, Gama CS, Ceresér KM, Yatham LN, Fries GR, Colpo G, de Lucena D, Kunz M, Gomes FA, Kapczinski F: Brain-derived neurotrophic factor as a state-marker of mood episodes in bipolar disorders: a systematic review and meta-regression analysis. J Psychiatr Res 2011, 45(8):995-1004.

43. Kenny MA, Williams JM: Treatment-resistant depressed patients show a good response to mindfulness-based cognitive therapy. Behav Res Ther 2007, 45:617-625.

44. Hamilton M: Development of a rating scale for primary depressive illness. Br J Soc Clin Psychol 1967, 6:278-296.

45. Young RC, Biggs JT, Ziegler VE, Meyer DA: A rating scale for mania: reliability, validity and sensitivity. Br J Psychiatry 1978, 133:429-435.

46. Spearing MK, Post RM, Leverich GS, Brandt D, Nolen W: Modification of the Clinical Global Impressions (CGI) scale for use in bipolar illness (BP): the CGI-BP. Psychiatry Res 1997, 73(3):159-171.

47. Hamilton M: The assessment of anxiety states by rating. Br J Med Psychol 1959, 32:50-55.

48. Stroop JR: Studies of interference in serial verbal reactions. J Exp Psychol 1935, 18:643-662.

49. Rosa AR, Sanchez-Moreno J, Martinez-Aran A, Salamero M, Torrent C, Reinares M, Comes M, Clom F, Van Riel W, Ayuis-Mateos JL, Kapczinski F, Vieta E: Validity and reliability of the Functioning Assessment Short Test (FAST) in bipolar disorder. Clin Pract Epidemiol Ment Health 2007, 3:5.

50. Segal Z, Teasdale J, Williams M, Gemar M: The mindfulness-based cognitive therapy adherence scale: inter-rater reliability, adherence to protocol and treatment distinctiveness. Clin Psychol Psychother 2002, 9:131-138.

51. Colom F, Vieta E: Psychoeducation Manual for Bipolar Disorder. Cambridge: Cambridge University Press; 2007

52. Ramos-Brieva JCA: Validación de la versión castellana de la escala de Hamilton para la depresión. Actas Luso-Esp Neurol Psiquiatr 1986 14:324-334.

53. Conners CK: Conners' Continuous Performance Test: User's manual. North Tonawanda, NY: Multi-Health Systems; 1995.

54. Dyer FN: The Stroop phenomenon and its use in the study of perceptual, cognitive and response processes. Mem Cognit 1973, 1:106-120.

55. Wechsler D: Wechsler Memory Scale. 3rd edition. San Antonio, TX: Psychological Corporation; 1997.

56. Heaton RK: Wisconsin Card Sorting Test Manuel. Odessa (FL): Psychological Assessment Resource Inc; 1981.

57. Kerr SL, Neale JM: Emotion perception in schizophrenia: specific deficit or further evidence of generalized poor performance? J Abnorm Psychol 1993, 102:312-318. 
58. Baer RA, Smith GT, Hopkins J, Krietemeyer J, Toney L: Using self-report assessment methods to explore facets of mindfulness. Assessment 2006, 13(1):27-45.

59. Soler J, Tejedor R, Feliu-Soler A, Pascual JC, Cebolla A, Soriano J, Alvarez E, Perez V: Psychometric proprieties of Spanish version of Mindful Attention Awareness Scale (MAAS). Actas Esp Psiquiatr 2012, 40(1):19-26.

doi:10.1186/s12888-014-0215-x

Cite this article as: Lahera et al:: Mindfulness-based cognitive therapy versus psychoeducational intervention in bipolar outpatients with sub-threshold depressive symptoms: a randomized controlled trial. BMC Psychiatry 2014 14:215.

\section{Submit your next manuscript to BioMed Central and take full advantage of:}

- Convenient online submission

- Thorough peer review

- No space constraints or color figure charges

- Immediate publication on acceptance

- Inclusion in PubMed, CAS, Scopus and Google Scholar

- Research which is freely available for redistribution 